\title{
Effects of Renal Replacement Therapy on Renal Recovery after Acute Kidney Injury
}

\author{
Antoine G. Schneider Sean M. Bagshaw \\ Division of Critical Care Medicine, Faculty of Medicine and Dentistry, University of Alberta, Edmonton, Alta., Canada
}

\section{Key Words}

Acute kidney injury · Biocompatible membranes · Citrate anticoagulation · Renal replacement therapy

\begin{abstract}
Recovery of kidney function following an episode of acute kidney injury (AKI) is now acknowledged as a vital patientcentered outcome with clear health economic implications. In approximately 5-8\% of critically ill patients with more severe forms of $\mathrm{AKI}$, support with renal replacement therapy (RRT) is provided. Recent data have suggested that rates of RRT utilization in AKI are increasing. Despite advances in our understanding of how best to prescribe acute RRT in critically ill patients with AKI, additional aspects remain uncertain, predisposing to suboptimal delivery and variation in practice. Importantly, if, when, how, and by what principles we apply acute RRT for AKI are all treatment decision-related factors that are modifiable and may interact with recovery of kidney function. Limited data, mostly from observational studies and secondary analyses, have explored the specific association between acute RRT and recovery. Available data are not able to clarify whether providing any RRT in otherwise eligible patients with AKI impacts recovery. They are also unable to inform whether the timing or circumstance under which RRT is started impacts recovery. No studies have evaluated whether there is an optimal time to start RRT
\end{abstract}

to maximize the probability of recovery. Accumulated evidence, mostly derived from observational studies, suggests initial therapy in critically ill patients with AKI with continuous RRT, compared with intermittent modalities, improves the probability of recovery to dialysis independence. Evidence from high-quality randomized trials failed to show any association between delivered dose intensity of RRT and recovery. The use of biocompatible membranes for acute RRT may improve recovery in AKI; however, data are inconsistent. Limited data have evaluated the impact of membrane flux properties on recovery. Preliminary data have suggested that circuit anticoagulation with citrate, which results in a reduction in membrane-induced oxidative stress and leukocyte activation, may be associated with improved recovery; however, further corroborative data are needed. Additional evidence, ideally from randomized trials, is clearly needed to inform best practice in the delivery of acute RRT to optimize probability of recovery of kidney function for survivors of AKI.

(c) 2014 S. Karger AG, Base

Targeting Recovery from Acute Kidney Injury: Round Table Conference at the 19th International Conference on Continuous Renal Replacement Therapies (Manchester Grand Hyatt, San Diego, Calif., USA, March 2-3, 2014).

\section{KARGER}

E-Mail karger@karger.com

www.karger.com/nec
(C) 2014 S. Karger AG, Basel

$1660-2110 / 14 / 1274-0035 \$ 39.50 / 0$
Sean M. Bagshaw

Division of Critical Care Medicine, Faculty of Medicine and Dentistry University of Alberta, Clinical Sciences Building, 2-124E 8440-112 Street NW, Edmonton, AB T6G 2B7 (Canada)

E-Mail bagshaw@ualberta.ca 


\section{Introduction}

Acute kidney injury (AKI) is a rapidly increasing and widespread problem, in particular among hospitalized patients with acute illness [1]. Mortality associated with AKI is unacceptably high; however, largely through enhanced awareness [2], heightened monitoring [3], and improved processes of care [4], rates over the last decade have shown a modest decline [5]. This has naturally promoted a greater focus on the survivorship issues following an episode of AKI. Recovery of kidney function is increasingly recognized as an important short- and longterm patient-centered and health economic outcome.

Among those who survive their acute illness, recovery of kidney function may or may not occur [6]. The trajectory of kidney function may include complete recovery (i.e., a return to a level of function near premorbid levels), incomplete or partial recovery (i.e., a reduction in function relative to baseline but not requiring RRT), and nonrecovery (i.e., dialysis dependence). Partial or incomplete recovery may herald the development of new chronic kidney disease (CKD) if persistent loss of kidney function is significant. Studies have shown that the risk of incident or worsening CKD and end-stage kidney disease (ESKD) is higher among patients with AKI (whether RRT was acutely received or not) compared with matched nonAKI controls, even among those who may have had complete recovery from AKI [7-9]. New or worsened CKD or acceleration toward ESKD are major morbid events with significant downstream implications, including increased cardiovascular event risk, reduced health-related quality of life, and greater use of health services [10].

Ascertaining granular data on kidney function short of RRT dependence among survivors of AKI has been challenging. Accordingly, the majority of clinical studies have defined kidney recovery by whether patients remain dialysis dependent or not at various time points (i.e., hospital discharge, or 90-day or 1-year follow-up). This definition is sensible given its obvious importance for patients and because such data are easily captured and associated with patient level morbidity and system level resource utilization, as aforementioned. In large observational cohort studies, the rates of dialysis dependence amongst survivors of critical illness complicated by AKI have ranged between 13 and 29\% (generally assessed at the point of hospital discharge) [10-14]. Among studies with a longerterm follow-up, any episode of AKI, requiring RRT or not, appears to be associated with a severalfold higher risk for developing ESKD and dialysis dependence [11, 12, 1417]. Despite the pragmatism of using dialysis dependence to define recovery after AKI, this definition fails to consider the important modifying impact of new or worsened CKD and ongoing evidence of kidney damage (i.e., albuminuria) that fall short of needing RRT [18-20].

In a large cohort utilizing data from the United States Department of Veterans Affairs with detailed monitoring of kidney function for a 6-year period following critical illness, AKI was associated with a 4- to 6-fold increase in the risk of developing stage $4 \mathrm{CKD}$ [21]. These findings have been replicated in another large population-based cohort study [22] and summarized in a systematic review [23] showing an unequivocal association between an episode of AKI and development of CKD.

One of the challenges of the evaluation of long-term kidney recovery after AKI is the competing risk of death, recognizing the high mortality associated with critical illness complicated by AKI. In long-term surveillance of the critically ill patients enrolled in the Randomized Evaluation of Normal versus Augmented Levels of RRT (RENAL) study, those surviving to the 90-day follow-up were shown to be more likely to die within 1 year of the episode of AKI than develop ESKD requiring dialysis initiation [24].

In this brief review, we will specifically focus on how RRT-related factors may influence and modify kidney recovery following an episode of AKI.

\section{Impact of RRT on Recovery}

\section{Impact of a Decision to Initiate RRT on Renal \\ Recovery}

In critically ill patients with more severe forms of AKI, supportive therapy with RRT is often provided [14]. Lifethreatening complications such as severe hyperkalemia, refractory acidosis, and severe fluid overload resulting in respiratory failure associated with AKI can be readily corrected with RRT. In these circumstances, the need to provide RRT would appear unequivocal. However, for critically ill patients with severe AKI but who have not developed life-threatening complications, the optimal time and circumstances for starting RRT is uncertain. This has contributed to suboptimal quality of RRT delivery and a wide variation in practice across jurisdictions [25]. Some recent data have even suggested that RRT application could, in some situations, be deleterious and impair renal recovery $[26,27]$.

In a secondary analysis of the Stuivenberg Hospital Acute Renal Failure (SHARF) 4 study, the development of ESKD and dialysis dependence among survivors to
Schneider/Bagshaw 


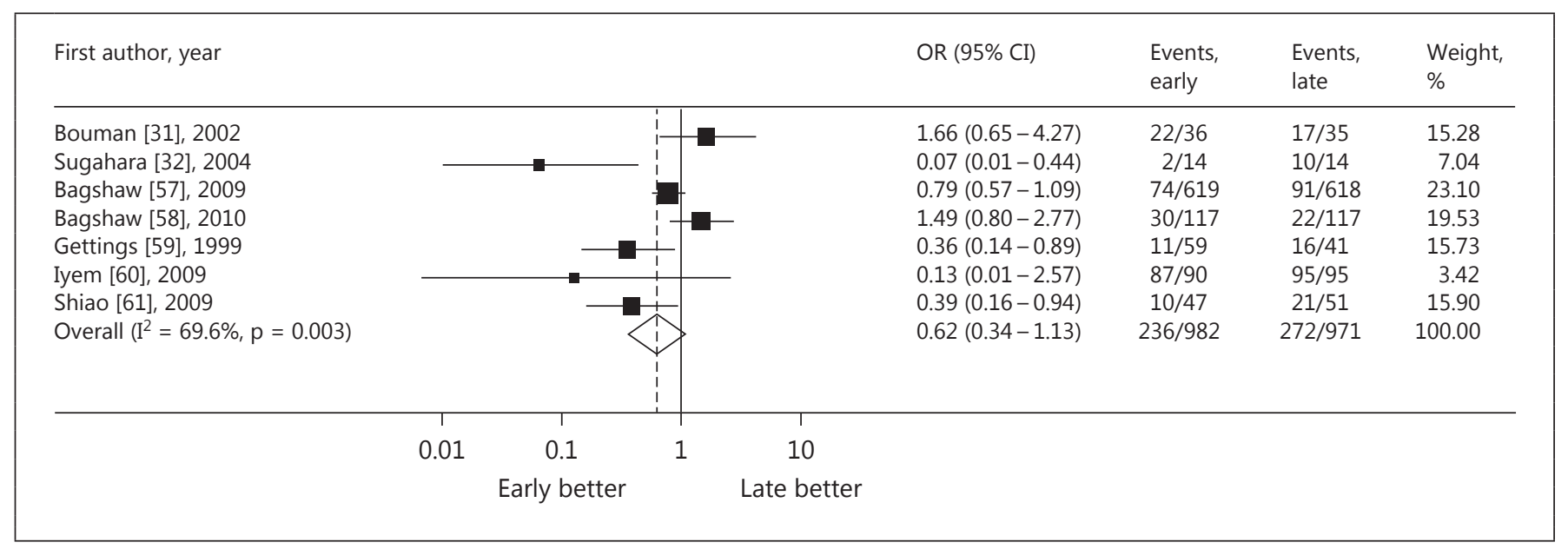

Fig. 1. Pooled analysis of clinical studies evaluating the association between timing of RRT initiation and recovery of kidney function (from Karvellas et al. [30]).

hospital discharge was found to be more common among RRT-treated compared with those treated more conservatively (no RRT; $24 \%$ for RRT vs. 9\% for no RRT) [27]. In this study, treatment allocation bias was not adequately adjusted for as RRT initiation was not standardized and left to the discretion of the treating clinician. This contributed to significant variation in the utilization of RRT and observed mortality across contributing sites. Similarly, a large multicenter French study utilizing a comprehensive clinical and administrative database evaluated the association between RRT utilization in AKI and outcome [26]. In multivariable-adjusted analysis, including propensity score matching for receipt of RRT, no differences in mortality were found between those with and without RRT. While the authors reported that $27.2 \%$ of RRT-treated survivors remained RRT dependent at discharge from the intensive care unit, there was no longerterm ascertainment of whether further recovery occurred in these patients or description of whether non-RRTtreated patients progressed to ESKD. Both of these studies remain prone to bias and residual confounding, and neither offered explanations for why selected patients with AKI and a high risk for mortality did not receive RRT.

Additional studies have clearly shown that, in the absence of consensus guidelines for when to optimally initiate RRT, RRT-treated patients remain fundamentally different from non-RRT-treated patients, which largely precludes any inferences from data outside of randomized trials $[28,29]$. This was evident in a recent secondary

Effects of RRT on Renal Recovery after AKI analysis of the Beginning and Ending Supportive Therapy for the Kidney (BEST-Kidney) study, a large international multicenter observational study of AKI [28]. In this analysis, the decision to provide RRT in severe AKI appeared largely predicated on treating clinicians' expectation of benefit. In close to $50 \%$ of the cases, RRT was withheld because of limitations of support or advanced age, where no benefit from RRT was expected. At the other end of the spectrum, RRT was also not provided to patients who appeared likely to undergo spontaneous recovery, such as those with adequate urinary output. Similar findings were made in another cohort [29] where limitations of medical therapy and perception of imminent renal recovery were the main reasons for not providing RRT. Unfortunately, these data collectively do not offer a clear view of whether the provision of any RRT in critically ill patients with AKI modifies the short- and longterm recovery of kidney function.

\section{Impact of RRT Timing on Renal Recovery}

There are limited data specifically evaluating the timing and circumstances of RRT initiation in AKI patients on recovery of kidney function. A recent systematic review focusing on RRT timing in critically ill patients with AKI suggested earlier RRT compared with late, delayed, or standard RRT was associated with a nonsignificant trend for improved recovery [odds ratio (OR) 0.62, 95\% confidence interval (CI) $0.34-1.13$; 7 studies; 1,953 patients] (fig. 1) [30]. However, the studies included in this pooled analysis had a high risk of bias due to issues of 
Table 1. Summary of selected studies evaluating the impact of initial RRT modality on renal recovery after AKI

\begin{tabular}{|c|c|c|c|c|c|c|}
\hline Mehta [38] & USA & $1991-1995$ & $\mathrm{MC}, \mathrm{RCT}$ & Mixed ICU & $\begin{array}{l}\mathrm{sCr} \text { return to } \\
\leq 177 \mu \mathrm{mol} / \mathrm{l}(2 \mathrm{mg} / \mathrm{dl})\end{array}$ & $\uparrow$ complete recovery with CRRT \\
\hline Bell [12] & Sweden & $1995-2004$ & $\mathrm{MC}, \mathrm{RC}$ & Mixed ICU & RRT free & $\downarrow$ ESKD with CRRT (8 vs. 17\%) \\
\hline Uchino $[40]$ & World & $2000-2001$ & $\mathrm{MC}, \mathrm{PC}$ & Mixed ICU & RRT free & $\downarrow$ DD with CRRT (11 vs. $35 \%)$ \\
\hline Jacka [54] & Canada & 2004 & SC, RC & Mixed ICU & RRT free & $\downarrow$ DD with CRRT (17 vs. 64\%) \\
\hline Andrikos [56] & Greece & 2008 & $\mathrm{MC}, \mathrm{PC}$ & Mixed ICU & RRT free & $\downarrow$ DD with CRRT (15 vs. $25 \%$ ) \\
\hline Wald [41] & Canada & 1996-2009 & $\mathrm{MC}, \mathrm{RC}$ & Mixed ICU & $\begin{array}{l}\text { RRT free conditional on } \\
\text { survival to } 90 \text { days }\end{array}$ & $\downarrow$ ESKD with CRRT (16 vs. $26 \%$ ) \\
\hline
\end{tabular}

$\mathrm{SC}=$ Single center $\mathrm{MC}=$ multicenter $\mathrm{PC}=$ prospective cohort $\mathrm{RC}=$ retrospective cohort; $\mathrm{sCr}=$ serum creatinine; $\mathrm{IHD}=$ intermit tent hemodialysis; ICU = intensive care unit; DD = dialysis dependence; RIFLE = Risk, Injury, Failure, Loss of Kidney Function and End-Stage Kidney Disease Classification.

study design, case mix, definitions for 'timing', and variation in outcome ascertainment. Few randomized trials have evaluated early versus later RRT initiation [30-32]. The only high-quality trial randomly allocated 106 mechanically ventilated patients with AKI to three strategies of RRT (early high volume, early low volume, or late low volume) [31]. The trial found no significant differences in mortality or recovery of kidney function between the treatment strategies. Moreover, only $1.6 \%$ of survivors remained RRT dependent, limiting any robust comparison of timing on recovery.

Fluid accumulation in critically ill patients, in particular those with AKI, has clearly been associated with increased mortality and may be treated with RRT [33-35]. Recently, a small retrospective cohort study suggested excess fluid accumulation prior to starting RRT in critically ill patients with AKI was associated with a reduced likelihood of recovery [36]. These data imply that fluid accumulation may represent an important trigger for when to start RRT. Moreover, RRT may be one important element in a spectrum of options to mitigate excess fluid accumulation and overload in critically ill patients, independent of AKI.

The timing and circumstances under which RRT is initiated is clearly modifiable; however, high-quality data showing their association with recovery are lacking. Randomized trials on this issue are fortunately ongoing [37].

\section{Impact of RRT Modality on Renal Recovery}

RRT can be provided as continuous (CRRT) or intermittent (IRRT) therapy. The first evidence to suggest there may be an important difference in the rate of recovery was derived from a randomized clinical trial (RCT) comparing CRRT and IRRT [38]. This study found on intention-to-treat analysis that a lower number of surviving CRRT-treated patients were discharged with residual kidney dysfunction compared with IRRT-treated patients ( 4 vs. $17 \%$, respectively); however, baseline case mix differences between groups and treatment crossover present challenges for strong inferences.

Coherent with these data are several large cohort studies showing rates of recovery to dialysis independence are lower for critically ill patients initially treated with CRRT compared with IRRT (table 1) [12,39-41]. While neither RENAL nor the Acute Renal Failure Trial Network Study (ATN) showed any survival or renal recovery differences with dose intensity of RRT, there were striking differences between the two trials with respect to dialysis dependence at 28 days ( $45.2 \%$ for ATN vs. $13.3 \%$ for RENAL) or at the end of follow-up (24.6\% for ATN vs. $5.6 \%$ for RENAL). While a direct comparison of the treatment strategies used between the trials is obviously prone to bias and challenging, it does question whether, in part, the greater exposure to IRRT in ATN compared with exclusive treatment with CRRT in RENAL is a contributing factor [42]. Recently, a large population-based Canadian study of critically ill patients surviving to 90 days after an episode of AKI treated with RRT utilizing propensity matching for treatment allocation found initial treatment with CRRT compared with IRRT was associated with a significantly lower likelihood of ESKD and dialysis dependence both at 90 days (16 vs. $21 \%$ ) and during the
38 
long-term follow-up (21 vs. 27\%). Observations from two large, high-quality randomized trials provide further insight into the impact of RRT modality on renal recovery $[43,44]$.

A recent systematic review identified 50 unique studies reporting the rate of dialysis dependence among more than 6,500 survivors of AKI who received RRT for AKI (not including the recent study by Wald et al. [41]) [45]. In a pooled analysis, including both observational studies and randomized trials, IRRT as initial treatment modality was associated with a 1.7 times greater risk for dialysis dependence when compared with initial treatment with CRRT (OR 1.73; 95\% CI 1.35-1.68). This finding was robust across subgroups including those with CKD; however, it did not reach statistical significance when the analysis was restricted to RCTs (OR 1.73; 95\% CI $0.73-$ $1.68 ; n=7)$. Available RCTs were generally small, of lower quality, and had relatively few events for pooled analysis. In addition, observational studies showed evidence of treatment selection bias with IRRT-treated patients having lower illness severity, greater hemodynamic stability, and greater rates of CKD at baseline. Collectively, these data imply that among critically ill patients with AKI requiring RRT, initial therapy, at least with CRRT, may confer a higher likelihood of recovery to dialysis independence.

\section{Impact of RRT Dose/Intensity on Renal Recovery}

Although highly debated during the previous decade, the question of whether the delivered dose of RRT impacts survival among critically ill patients with AKI has largely been resolved following the publication of two high-quality RCTs $[43,44]$. In addition to showing no difference in survival, neither study found evidence that the RRT dose/intensity delivered affected recovery to dialysis independence.

\section{Impact of Membrane Type on Renal Recovery}

Selected studies have suggested the biocompatibility of the filter membrane used for IRRT in patients with AKI may influence recovery of kidney function [46, 47]; however, these findings have not been universal or consistent $[48,49]$. In a small controlled trial of 52 patients with AKI, RRT utilizing cuprophane membranes (bioincompatible) elicited greater activation of complement (serum C3a) and oxidative stress (leukotriene B4) when compared to RRT with polyacrylonitrile membranes (biocompatible) [47]. This activation and alternation in immune function was believed to contribute to delayed or reduced likelihood of recovery. Indeed, in this trial, the group receiving

Effects of RRT on Renal Recovery after AKI
RRT with cuprophane membranes required more RRT sessions (301 vs. 222 ) and was more likely to remain dialysis dependent 3 weeks after its initiation (33 vs. 25\%). Among those weaned from RRT, the cuprophane-treated group required a longer duration until recovery (22 vs. 15 days) and was less likely to have normalized serum creatinine levels at 3 months (66 vs. $88 \%$ ). These findings were supported by another small randomized trial of 72 patients with AKI allocated to receive RRT with either a cuprophane or polymethylmethacrylate membrane [46]. Those receiving RRT with cuprophane membranes had lower adjusted rates of recovery to dialysis independence ( 37 vs. $62 \%, \mathrm{p}=0.04$ ). Among those recovering, use of a cuprophane membrane was associated with longer duration of RRT support ( 17 vs. 5 sessions, $p=0.02$ ). Additional randomized trials and a systematic review (10 studies, 1,100 patients) found no clinical or statistical differences in recovery to dialysis independence by membrane compatibility $[48,49]$.

Fewer data have focused specifically on the membrane flux properties and recovery of kidney function in AKI. In a small, randomized trial of 72 critically ill patients with AKI treated with IRRT, the use of high-flux (F12) compared with low-flux (F6) biocompatible membranes showed no difference in the rates of survival and recovery of kidney function to dialysis independence [50].

\section{Impact of Anticoagulation Type on Renal Recovery}

There is a paucity of data evaluating the impact of the choice of the anticoagulation type on the course of AKI and recovery of kidney function. Studies have suggested that use of citrate as an anticoagulant may have added pleiotropic effects beyond regional anticoagulation of the extracorporeal circuit. One small, randomized trial in 20 critically ill patients receiving CRRT for AKI compared the effect of regional citrate and heparin anticoagulation on measures of systemic oxidative stress and inflammation [51]. Citrate reduced prefilter myeloperoxidase and interleukin-8 levels compared with heparin. While heparin was associated with a reduction in tumor necrosis factor- $\alpha$, prefilter myeloperoxidase levels were significantly higher compared with citrate. Similarly, heparin has been shown to activate complement and induce neutrophil degranulation in the extracorporeal circuit to a greater extent when compared with citrate [52]. Whether these observations are also impacted by citrate being associated with longer filter survival and reduced episodes of bleeding and need for transfusion is plausible but remains uncertain [53]. 


\section{Conclusion}

Recovery of kidney function after AKI is a vital patient-centered outcome with downstream health service and economic implications. In critical care settings, severe AKI is commonly treated with RRT and its utilization appears to be expanding. Importantly, the broad principles for how RRT is provided (i.e., timing, dose, modality, circuit characteristics, and anticoagulation) to critically ill patients are all modifiable and hence susceptible to practice variation. This would imply that treatment applied within current best practice may improve quality of care and outcomes; however, there is a relative paucity of sufficiently high quality evidence investigating the association between RRT and recovery of kidney function among survivors of AKI. Available data suggest initial therapy with CRRT, and use of biocompatible membranes and citrate anticoagulation may have some benefit to improve recovery; however, if and when to provide RRT still needs better clarification, and further studies focusing on renal recovery as an outcome are urgently needed.

\section{Acknowledgments}

Dr. Bagshaw holds a Canada Research Chair in Critical Care Nephrology and is a Clinical Investigator supported by Alberta Innovates - Health Solutions.

\section{References}

1 Rewa O, Bagshaw SM: Acute kidney injury epidemiology, outcomes and economics. Nat Rev Nephrol 2014;10:193-207.

-2 Li PK, Burdmann EA, Mehta RL; World Kidney Day Steering Committee: Acute kidney injury: global health alert. Kidney Int 2013;83: 372-376.

-3 Selby NM, Crowley L, Fluck RJ, et al: Use of electronic results reporting to diagnose and monitor AKI in hospitalized patients. Clin J Am Soc Nephrol 2012;7:533-540.

4 Goldstein SL, Kirkendall E, Nguyen H, et al: Electronic health record identification of nephrotoxin exposure and associated acute kidney injury. Pediatrics 2013;132:e756e767.

5 Bagshaw SM, George C, Bellomo R: Changes in the incidence and outcome for early acute kidney injury in a cohort of Australian intensive care units. Crit Care 2007;11:R68.

6 Bellomo R, Ronco C, Kellum JA, Mehta RL, Palevsky P: Acute renal failure - definition outcome measures, animal models, fluid therapy and information technology needs: the Second International Consensus Conference of the Acute Dialysis Quality Initiative (ADQI) Group. Crit Care 2004;8:R204-R212.

7 Jones J, Holmen J, De Graauw J, Jovanovich A, Thornton S, Chonchol M: Association of complete recovery from acute kidney injury with incident CKD stage 3 and all-cause mortality. Am J Kidney Dis 2012;60:402-408.

$\checkmark 8$ Wald R, Quinn RR, Adhikari NK, et al: Risk of chronic dialysis and death following acute kidney injury. Am J Med 2012;125:585-593.

9 Wald R, Quinn RR, Luo J, et al: Chronic dialysis and death among survivors of acute kidney injury requiring dialysis. JAMA 2009;302: 1179-1185.

10 Korkeila M, Ruokonen E, Takala J: Costs of care, long-term prognosis and quality of life in patients requiring renal replacement ther- 19 Stevens PE, Levin A: Evaluation and manageapy during intensive care. Intensive Care Med 2000;26:1824-1831.

11 Bagshaw SM, Laupland KB, Doig CJ, et al: Prognosis for long-term survival and renal recovery in critically ill patients with severe acute renal failure: a population-based study. Crit Care 2005;9:R700-R709.

12 Bell M, Granath F, Schon S, Ekbom A, Martling CR: Continuous renal replacement therapy is associated with less chronic renal failure than intermittent haemodialysis after acute renal failure. Intensive Care Med 2007; 33:773-780.

13 Silvester W, Bellomo R, Cole L: Epidemiology, management, and outcome of severe acute renal failure of critical illness in Australia. Crit Care Med 2001;29:1910-1915.

14 Uchino S, Kellum JA, Bellomo R, et al: Acute renal failure in critically ill patients: a multinational, multicenter study. JAMA 2005;294: 813-818.

15 Hsu CY, Chertow GM, McCulloch CE, Fan D, Ordonez JD, Go AS: Nonrecovery of kidney function and death after acute on chronic renal failure. Clin J Am Soc Nephrol 2009;4: 891-898.

16 Lo LJ, Go AS, Chertow GM, et al: Dialysisrequiring acute renal failure increases the risk of progressive chronic kidney disease. Kidney Int 2009;76:893-899.

17 Wu VC, Huang TM, Lai CF, et al: Acute-onchronic kidney injury at hospital discharge is associated with long-term dialysis and mortality. Kidney Int 2011;80:1222-1230.

18 Gansevoort RT, Matsushita K, van der Velde $\mathrm{M}$, et al: Lower estimated GFR and higher albuminuria are associated with adverse kidney outcomes. A collaborative meta-analysis of general and high-risk population cohorts. Kidney Int 2011;80:93-104. ment of chronic kidney disease: synopsis of the kidney disease: improving global outcomes 2012 clinical practice guideline. Ann Intern Med 2013;158:825-830.

20 van der Velde M, Matsushita K, Coresh J, et al: Lower estimated glomerular filtration rate and higher albuminuria are associated with all-cause and cardiovascular mortality. A collaborative meta-analysis of high-risk population cohorts. Kidney Int 2011;79:13411352.

21 Amdur RL, Chawla LS, Amodeo S, Kimmel PL, Palant CE: Outcomes following diagnosis of acute renal failure in U.S. veterans: focus on acute tubular necrosis. Kidney Int 2009;76: 1089-1097.

22 James MT, Hemmelgarn BR, Wiebe N, et al: Glomerular filtration rate, proteinuria, and the incidence and consequences of acute kidney injury: a cohort study. Lancet 2010;376: 2096-2103.

23 Coca SG, Singanamala S, Parikh CR: Chronic kidney disease after acute kidney injury: a systematic review and meta-analysis. Kidney Int 2012;81:442-448.

24 Gallagher M, Cass A, Bellomo R, et al: Longterm survival and dialysis dependency following acute kidney injury in intensive care: extended follow-up of a randomized controlled trial. PLoS Med 2014;11:e1001601.

25 Ricci Z, Ronco C, D’Amico G, et al: Practice patterns in the management of acute renal failure in the critically ill patient: an international survey. Nephrol Dial Transplant 2006; 21:690-696.

26 Clec'h C, Gonzalez F, Lautrette A, et al: Multiple-center evaluation of mortality associated with acute kidney injury in critically ill patients: a competing risks analysis. Crit Care 2011;15:R128. 
27 Elseviers MM, Lins RL, Van der Niepen P, et al: Renal replacement therapy is an independent risk factor for mortality in critically ill patients with acute kidney injury. Crit Care 2010;14:R221.

28 Bagshaw SM, Uchino S, Kellum J, et al: Association between renal replacement therapy in critically ill patients with severe acute kidney injury and mortality. J Crit Care 2013;28: 1011-1018.

29 Schneider AG, Uchino S, Bellomo R: Severe acute kidney injury not treated with renal replacement therapy: characteristics and outcome. Nephrol Dial Transplant 2012;27:947952.

-30 Karvellas CJ, Farhat MR, Sajjad I, et al: A comparison of early versus late initiation of renal replacement therapy in critically ill patients with acute kidney injury: a systematic review and meta-analysis. Crit Care 2011;15:R72.

- 31 Bouman CS, Oudemans-Van Straaten HM, Tijssen JG, Zandstra DF, Kesecioglu J: Effects of early high-volume continuous venovenous hemofiltration on survival and recovery of renal function in intensive care patients with acute renal failure: a prospective, randomized trial. Crit Care Med 2002;30:2205-2211.

-32 Sugahara S, Suzuki H: Early start on continuous hemodialysis therapy improves survival rate in patients with acute renal failure following coronary bypass surgery. Hemodial Int 2004;8:320-325.

33 Bouchard J, Soroko SB, Chertow GM, et al: Fluid accumulation, survival and recovery of kidney function in critically ill patients with acute kidney injury. Kidney Int 2009;76:422427.

-34 Vaara ST, Korhonen AM, Kaukonen KM, et al: Fluid overload is associated with an increased risk for 90-day mortality in critically ill patients with renal replacement therapy: data from the prospective FINNAKI study. Crit Care 2012;16:R197.

35 Grams ME, Estrella MM, Coresh J, Brower RG, Liu KD: Fluid balance, diuretic use, and mortality in acute kidney injury. Clin J Am Soc Nephrol 2011;6:966-973.

- 36 Heung M, Wolfgram DF, Kommareddi M, $\mathrm{Hu}$ Y, Song PX, Ojo AO: Fluid overload at initiation of renal replacement therapy is associated with lack of renal recovery in patients with acute kidney injury. Nephrol Dial Transplant 2012;27:956-961.

37 Smith OM, Wald R, Adhikari NK, et al: Standard versus accelerated initiation of renal replacement therapy in acute kidney injury (STARRT-AKI): study protocol for a randomized controlled trial. Trials 2013;14:320.

38 Mehta RL, McDonald B, Gabbai FB, et al: A randomized clinical trial of continuous versus intermittent dialysis for acute renal failure. Kidney Int 2001;60:1154-1163.
39 Lin YF, Ko WJ, Chu TS, et al: The 90-day mortality and the subsequent renal recovery in critically ill surgical patients requiring acute renal replacement therapy. Am J Surg 2009; 198:325-332.

40 Uchino S, Bellomo R, Kellum JA, et al: Patient and kidney survival by dialysis modality in critically ill patients with acute kidney injury. Int J Artif Organs 2007;30:281-292.

41 Wald R, Shariff SZ, Adhikari NK, et al: The association between renal replacement therapy modality and long-term outcomes among critically ill adults with acute kidney injury: a retrospective cohort study. Crit Care Med 2014; $42: 868-877$

42 Glassford NJ, Bellomo R: Acute kidney injury: how can we facilitate recovery? Curr Opin Crit Care 2011;17:562-568.

43 RENAL Replacement Therapy Study Investigators, Bellomo R, Cass A, Cole L, et al: Intensity of continuous renal-replacement therapy in critically ill patients. N Engl J Med 2009; 361:1627-1638.

44 VA/NIH Acute Renal Failure Trial Network, Palevsky PM, Zhang JH, O'Connor TZ, et al: Intensity of renal support in critically ill patients with acute kidney injury. N Engl J Med 2008;359:7-20.

45 Schneider AG, Bellomo R, Bagshaw SM, et al: Choice of renal replacement therapy modality and dialysis dependence after acute kidney injury: a systematic review and meta-analysis. Intensive Care Med 2013;39:987-997.

46 Hakim RM, Wingard RL, Parker RA: Effect of the dialysis membrane in the treatment of patients with acute renal failure. $\mathrm{N}$ Engl J Med 1994;331:1338-1342.

47 Schiffl H, Lang SM, Konig A, Strasser T, Haider MC, Held E: Biocompatible membranes in acute renal failure: prospective case-controlled study. Lancet 1994;344:570-572.

48 Alonso A, Lau J, Jaber BL: Biocompatible hemodialysis membranes for acute renal failure. Cochrane Database Syst Rev 2008;1:CD005283.

49 Gastaldello K, Melot C, Kahn RJ, Vanherweghem JL, Vincent JL, Tielemans C: Comparison of cellulose diacetate and polysulfone membranes in the outcome of acute renal failure. A prospective randomized study. Nephrol Dial Transplant 2000;15:224-230.

-50 Ponikvar JB, Rus RR, Kenda RB, Bren AF, Ponikvar RR: Low-flux versus high-flux synthetic dialysis membrane in acute renal failure: prospective randomized study. Artif Organs 2001;25:946-950.

51 Tiranathanagul K, Jearnsujitwimol O, Susantitaphong $\mathrm{P}$, et al: Regional citrate anticoagulation reduces polymorphonuclear cell degranulation in critically ill patients treated with continuous venovenous hemofiltration. Ther Apher Dial 2011;15:556-564.
52 Schilder L, Nurmohamed SA, ter Wee PM, et al: Citrate confers less filter-induced complement activation and neutrophil degranulation than heparin when used for anticoagulation during continuous venovenous haemofiltration in critically ill patients. BMC Nephrol 2014;15:19.

53 Wu MY, Hsu YH, Bai CH, Lin YF, Wu CH, Tam KW: Regional citrate versus heparin anticoagulation for continuous renal replacement therapy: a meta-analysis of randomized controlled trials. Am J Kidney Dis 2012;59: 810-818.

54 Jacka MJ, Ivancinova X, Gibney RT: Continuous renal replacement therapy improves renal recovery from acute renal failure. Can J Anaesth 2005;52:327-332.

55 Cartin-Ceba R, Haugen EN, Iscimen R, Trillo-Alvarez C, Juncos L, Gajic O: Evaluation of 'Loss' and 'End stage renal disease' after acute kidney injury defined by the Risk, Injury, Failure, Loss and ESRD classification in critically ill patients. Intensive Care Med 2009;35: 2087-2095.

56 Andrikos E, Tseke P, Balafa O, et al: Epidemiology of acute renal failure in ICUs: a multicenter prospective study. Blood Purif 2009;28: 239-244.

57 Bagshaw SM, Uchino S, Bellomo R, Morimatsu H, Morgera S, Schetz M, Tan I, Bouman C, Macedo E, Gibney N, Tolwani A, Oudemansvan Straaten HM, Ronco C, Kellum JA: Timing of renal replacement therapy and clinical outcomes in critically ill patients with severe acute kidney injury. J Crit Care 2009;24:129140.

58 Bagshaw SM, Wald R, Barton J, Burns KE, Friedrich JO, House AA, James MT, Levin A, Moist L, Pannu N, Stollery DE, Walsh MW: Clinical factors associated with initiation of renal replacement therapy in critically ill patients with acute kidney injury-a prospective multicenter observational study. J Crit Care 2012;27:268-275.

59 Gettings LG, Reynolds HN, Scalea T: Outcome in post-traumatic acute renal failure when continuous renal replacement therapy is applied early vs. late. Intensive Care Med 1999;25:805-813.

60 Iyem H, Tavli M, Akcicek F, Buket S: Importance of early dialysis for acute renal failure after an open-heart surgery. Hemodial Int 2009;13:55-61.

-61 Shiao CC, Wu VC, Li WY, Lin YF, Hu FC, Young GH, Kuo CC, Kao TW, Huang DM, Chen YM, Tsai PR, Lin SL, Chou NK, Lin TH, Yeh YC, Wang CH, Chou A, Ko WJ, Wu KD: Late initiation of renal replacement therapy is associated with worse outcomes in acute kidney injury after major abdominal surgery. Crit Care 2009;13:R171.
Effects of RRT on Renal Recovery after AKI
Nephron Clin Pract 2014;127:35-41 